\title{
Towards an Optimal Interest Point Detector for Measurements in Ultrasound Images
}

\author{
Martin Zukal, Radek Beneš, Petr Číka, and Kamil Říha \\ Department of Telecommunications, Brno University of Technology, Technická 12, 61600 Brno, Czech Republic \\ martin.zukal@phd.feec.vutbr.cz
}

\begin{abstract}
This paper focuses on the comparison of different interest point detectors and their utilization for measurements in ultrasound (US) images. Certain medical examinations are based on speckle tracking which strongly relies on features that can be reliably tracked frame to frame. Only significant features (interest points) resistant to noise and brightness changes within US images are suitable for accurate long-lasting tracking. We compare three interest point detectors - Harris-Laplace, Difference of Gaussian (DoG) and Fast Hessian - and identify the most suitable one for use in US images on the basis of an objective criterion. Repeatability rate is assumed to be an objective quality measure for comparison. We have measured repeatability in images corrupted by different types of noise (speckle noise, Gaussian noise) and for changes in brightness. The Harris-Laplace detector outperformed its competitors and seems to be a sound option when choosing a suitable interest point detector for US images. However, it has to be noted that Fast Hessian and DoG detectors achieved better results in terms of processing speed.
\end{abstract}

Keywords: B-mode ultrasound, Gaussian noise, interest point detector, repeatability rate, speckle noise, speckle tracking.

\section{INTRODUCTION}

$\mathrm{M}$ EDICAL EXAMINATIONS have changed a lot over the past years. Nowadays, a standard examination is often accompanied by additional examinations that use some of the modern medical imaging techniques [1]. These techniques rely on signals that are beyond the perception of human beings. An example of such a signal is ultrasound that lies above the human hearing limits.

Medical sonography has recently become one of the most important and most popular medical imaging techniques. It is based on ultrasound wave pulses. These pulses are generated by a transducer and travel through the human body reflecting on the inhomogeneous interfaces of two tissues. The transducer receives the reflected pulses (echoes) and conveys them to a sonographic system that displays an appropriate output generated on the basis of parameters of the echoes.

Several different modes of ultrasound are used in medical imaging such as A-mode (one-dimensional output), B-mode, M-mode (visualization of motion of organ structures, video in ultrasound), Doppler mode (blood flow visualization) or 3D ultrasound. We will focus on B-mode ultrasound that generates $2 \mathrm{D}$ images in what follows.

The examination with ultrasound has many advantages including a non-invasive principle, portability of a scanner, fast scanning and low price in comparison to other methods. Conversely, there are certain drawbacks some of which are low resolution of the images, speckle noise, scattering and presence of artifacts [2]. Especially the presence of speckle noise [3] in images complicates their further processing.

This paper focuses on the analysis and performance comparison of interest point detectors for ultrasound images. Detection of interest points is a very important issue in many examinations based on speckle tracking [4]. Speckle track- ing is a technique that makes use of tracking of interest points to measure velocities and deformation parameters of tissues. These examinations are often performed on video-sequences and the diagnosis is based on the movement of interest points in time. Examples cover generally all dynamic processes that can be examined with ultrasound. They include tracking of movement of heart wall, arterial wall, emptying of bladder or stomach to name a few. Not only movement, but also volume of organs can be measured and subsequently used for various medical examinations. Jayasree et al. [5] studied correlations between certain cardiovascular diseases and photoplethysmographic (PPG) waveform. Hlimonenko et al. [6] employ the PPG waveform to find the correlation between the elastic properties of vascular tree and age. Similar studies can be performed with waveform obtained from large arteries using speckle tracking in US video sequences. The boundaries of the examined object have to be described by a set of very robust interest points that will be resistant to the level of noise present in US images in order to obtain accurate results.

Many examples of medical examinations based on speckle tracking are summarized in the related work chapter. One of the simplest examples is the continual measurement of lumen diameter. The lumen is represented as a set of interest points on its wall. The interest points form a circle in the transversal section and two parallel lines in the longitudinal sections. The tracked interest points must be invariant to US noise as much as possible for a long-lasting measurement with high accuracy. The aim of this paper is to verify capabilities of state-ofthe-art interest point detectors and assess their robustness with respect to noise level and changes in brightness according to an objective criterion.

The exact terminology as well as the definition of interest point differs from author to author [7]. Some authors use the 
MEASUREMENT SCIENCE REVIEW, Volume 13, No. 6, 2013

term interest points, other authors use terms like salient points or local features but the meaning is the same. Generally, an interest point is a location in an image with high information content which is stable under varying viewing conditions and/or image degradations. Typical examples of these features are corners, junctions, dark blobs on light background, etc.

As there are no comparisons of suitability of different interest point detectors for ultrasound image processing available we implemented and tested three state-of-the-art detectors. Specifically, we tested the Harris-Laplace detector, the Fast Hessian detector and the Difference of Gaussian detector. These detectors will be described in detail in section 2 .

All detectors were tested on a database of ultrasound images of artery which contains 84 images acquired under varying conditions. Repeatability rate was used to evaluate the performance of each detector. We evaluated repeatability rate for images that were artificially corrupted by noise of different types and with changing variance.

\subsection{Related work}

There are certain medical examinations that analyze either movement or volume of organs in an ultrasound image sequence like estimation of ejection-fraction of heart or volume (area) of heart over time. Such examinations require sequences of images acquired in time and localized boundaries in the first frame. These boundaries are represented by a set of landmarks or visual features [8] in some approaches. For instance, the approach used by McEachen and Duncan [9] uses landmarks followed by a sequence of images. Some methods are based on optical flow, for example by Mailloux [10] and Friedland and Adams [11]. This method is also used for the measurement of longitudinal motion of the vessel wall $[12,13]$. Giachetti used the best features of the active contour and combined them with optical flow methods [14].

Similar principle can be used for continual measurement of arterial diameter in transversal section $[15,16,17]$. This examination is applicable to measurements of Endotheliumdependent vasoreactivity (EDV) and arterial compliance. Such measurements are important because abnormal EDV is strongly linked to atherosclerosis [18].

Two-dimensional tracking of the vessel wall movement can be also performed in the longitudinal section [12]. This examination might improve the ability to detect early abnormalities in vessel wall properties. Increased knowledge of the longitudinal movement in the arterial wall may provide new information not only on the mechanical properties of arteries, but also on the mechanical forces [19]. Similar measurement can be performed with many other organs, for example for measurement of gastric emptying [18], or bladder emptying [20].

Many interest point detectors have been proposed in literature over the last four decades. A thorough survey discussing great majority of them was published in [7], we therefore limited ourselves to highlighting few of them in this chapter. Probably the largest group of methods for interest point detec- tion is based on the differential approach. These algorithms detect the interest points on the basis of first or second order derivatives of the image function. Examples of algorithms belonging to this group are all the three algorithms described in section 2.

There are also other methods that follow completely different approaches. The wavelet-based salient point detector [21] that uses a multiresolution analysis can be mentioned as one of them. Gaussian filters used in scale-space analysis (described later on) smooth the image which results in loss of details. The details in the image are crucial in all speckle tracking methods. This suggests that the wavelet-based salient point detector could prove useful in ultrasound image processing. A completely different approach is adopted in [22] where the authors consider the image as a realisation of a zero mean Gaussian random process and the salient points are defined as rare events that are sparsely distributed under a stochastic model of the image. The local image structure is considered as an interest point if the likelihood estimation under a Brownian image model [23] is minimal. As the ultrasound images do not contain extremely distinct structures, the suitability of this approach is questionable, however, possible usefulness for ultrasound image processing of such a detector would have to be verified experimentally.

The need to evaluate these algorithms objectively appeared with the growing number of detectors. A methodology to evaluate these algorithms was proposed by Schmid et al. in [24]. They identified two crucial properties of interest point detectors in their work. These are the information content of the interest point and stability. The information content expresses the variability of the neighborhood of a point and is closely related to entropy. Stability describes the reliability of the algorithm to reproduce the same point in a modified image. Scale changes, rotations and changes of 3D viewpoint can be found among common modifications. Stability of an algorithm can be described by a measure called repeatability rate [24]. A number of studies comparing different interest point detectors have been published. Mikolajczyk and Schmid compare scale and affine invariant interest point detectors (including the Harris-Laplace and Hessian detector) in [25]. They focus on evaluation of the detectors in terms of scale change and change of viewpoint but they do not consider brightness change. [24] is the only study that assesses the dependence of repeatability rate on change of brightness. One of the detectors that is evaluated in that study is the Harris detector which is the basis of the Harris-Laplace detector used in our work. As we deal with modifications that do not change scale of the image it can be expected that the results of the Harris and Harris-Laplace algorithm will be similar in terms of change of brightness. An evaluation of the Harris-Laplace, Fast Hessian and DoG detectors with respect to change of brightness was performed in [26]. The performance of interest point detectors for tracking purposes in image sequence was evaluated in [27]. 


\section{INTEREST POINT DETECTORS}

In this section, the three tested detectors are described in detail. As we deal with scale-invariant interest point detectors, it is necessary to make a preliminary note on the scale-space representation of an image.

\subsection{Scale-Space Representation}

The scale-space representation [28] provides a way to describe the image on multiple scales (when taken to the limit it considers all possible scales). It has been shown that the Gaussian kernels and Gaussian derivatives can be effectively used to build the scale-space.

The scale-space function in point $\mathbf{x}=(x, y)$ is a convolution of the image function and a Gaussian kernel

$$
L(\mathbf{x}, \sigma)=G(\mathbf{x}, \sigma) * I(\mathbf{x}),
$$

where $I(\mathbf{x})$ denotes the image function. $G(\mathbf{x}, \sigma)$ represents the Gaussian kernel

$$
G(\mathbf{x}, \sigma)=\frac{1}{2 \pi \sigma^{2}} \mathrm{e}^{-\left(x^{2}+y^{2}\right) / 2 \sigma^{2}}
$$

with scale $\sigma$

The partial derivative (in $a$ direction) of the scale-space can be computed either by differentiating directly the scale-space function or as a convolution of the original image with the derivative of Gaussian kernel

$$
L_{a}(\mathbf{x}, \sigma)=\frac{\partial}{\partial a} L(\mathbf{x}, \sigma)=\frac{\partial}{\partial a} G(\mathbf{x}, \sigma) * I(\mathbf{x}) .
$$

\subsection{Harris-Laplace detector}

The corner detector [29] proposed by C.Harris is not scale invariant and its use is therefore rather limited. Nevertheless, a scale adapted modification of the Harris detector was proposed [25] and named the Harris-Laplace detector. Both, the Harris detector and the Harris-Laplace detector, detect interest points using the second order matrix which describes the structure of the local neighborhood of a point. The key difference is that the second order matrix is made invariant to scale in the Harris-Laplace detector. The modified second order matrix for point $\mathbf{x}=(x, y)$ is defined as

$M\left(\mathbf{x}, \sigma_{I}, \sigma_{D}\right)=\sigma_{D}^{2} G\left(\mathbf{x}, \sigma_{I}\right) *\left[\begin{array}{cc}L_{x}^{2}\left(\mathbf{x}, \sigma_{D}\right) & L_{x} L_{y}\left(\mathbf{x}, \sigma_{D}\right) \\ L_{x} L_{y}\left(\mathbf{x}, \sigma_{D}\right) & L_{y}^{2}\left(\mathbf{x}, \sigma_{D}\right)\end{array}\right]$

The idea is to average the local derivatives computed with Gaussian kernels of the scale $\sigma_{D}$ (differentiation scale) in the neighborhood of the point. The average can be effectively computed by means of convolution with a Gaussian window $G$ of the scale $\sigma_{I}$ (integration scale).

In the first step of the algorithm, candidate points are localized as maxima of the so called cornerness $H$

$$
H=\operatorname{det} M\left(\mathbf{x}, \sigma_{I}, \sigma_{D}\right)-k\left(\operatorname{trace} M\left(\mathbf{x}, \sigma_{I}, \sigma_{D}\right)\right)^{2},
$$

where $\operatorname{det} M\left(\mathbf{x}, \sigma_{I}, \sigma_{D}\right)=L_{x}^{2} L_{y}^{2}-\left(L_{x} L_{y}\right)^{2}$, trace $M\left(\mathbf{x}, \sigma_{I}, \sigma_{D}\right)=L_{x}^{2}+L_{y}^{2}$ and $k$ is a constant.

Only points for which the Laplacian-of-Gaussian

$$
\left|\operatorname{LoG}\left(\mathbf{x}, \sigma_{n}\right)\right|=\sigma_{n}^{2}\left|L_{x x}\left(\mathbf{x}, \sigma_{n}\right)+L_{y y}\left(\mathbf{x}, \sigma_{n}\right)\right|,
$$

reaches a maximum are selected as interest points in the second step. $L_{x x}(\mathbf{x}, \sigma)$ denotes the second order derivative of the scale-space function and it is computed as the convolution of the image $I$ in point $\mathbf{x}$ and the second order derivative of the Gaussian kernel in $x$ direction:

$$
L_{x x}(\mathbf{x}, \sigma)=I(\mathbf{x}) * \frac{\partial^{2}}{\partial x^{2}} G(\mathbf{x}, \sigma) .
$$

$L_{y y}(\mathbf{x}, \sigma)$ is calculated in the same way but the second order derivative of the Gaussian kernel in $y$ direction $\left(\frac{\partial^{2}}{\partial y^{2}} G(\mathbf{x}, \sigma)\right)$ is used. The detailed description of the algorithm is described in [25].

\subsection{Fast Hessian detector}

The Fast Hessian detector [30] differs from the Harris detector in the matrix used to detect the interest points. It is based on the Hessian matrix which can be described in the following way

$$
H(\mathbf{x}, \sigma)=\left[\begin{array}{ll}
L_{x x}(\mathbf{x}, \sigma) & L_{x y}(\mathbf{x}, \sigma) \\
L_{x y}(\mathbf{x}, \sigma) & L_{y y}(\mathbf{x}, \sigma)
\end{array}\right],
$$

where $L_{x x}(\mathbf{x}, \sigma)$ and $L_{y y}(\mathbf{x}, \sigma)$ again denote the second order derivatives of the scale-space function as defined in 7. $L_{x y}(\mathbf{x}, \sigma)$ is based on convolution with $\frac{\partial^{2}}{\partial y \partial x} G(\mathbf{x}, \sigma)$ and computed in accordance with 7 .

The Gaussian derivative has to be cropped and discretized in order to use it in calculations. The authors of the Fast Hessian detector proposed the use of the so called box filters that approximate the second order Gaussian derivatives. The advantage of the box filters is that they can be computed very fast when the integral images [31] are used. Instead of downscaling the image during the process of detection, it is possible to achieve the same effect by up-scaling the box filters. The complexity of computation of the response of the box filter is independent of its size [31] which results in even higher processing speed.

The determinant of the Hessian matrix is computed for every point in the image and the local maxima are detected similarly to DoG detector described in the following section.

\subsection{Difference of Gaussian detector}

Unlike the Harris-Laplace detector, the Difference of Gaussian (DoG) detector is a blob detector. Blobs are described as regions in the image that are brighter or darker than their surroundings.

DoG detector [32] cannot be described using the matrix equations as the previous two detectors because the detection 
is based on direct subtraction of neighboring scale-space functions. The DoG function is defined as

$$
D(\mathbf{x}, \sigma)=L(\mathbf{x}, k \sigma)-L(\mathbf{x}, \sigma)=(G(\mathbf{x}, k \sigma)-G(\mathbf{x}, \sigma)) * I(\mathbf{x}),
$$

where $k$ is a constant. $D(\mathbf{x}, \sigma)$ can be understood as an approximation of the scale normalized Laplacian of Gaussian.

The interest points are selected as the extrema of the DoG. The point is selected as an interest point only if it is larger or smaller than all its neighbors. The point is compared to its eight neighbors in the current scale and to nine neighbors in the scale above and nine neighbors in the scale below. Undertaking some additional steps is necessary in order to get the final set of interest points but this exceeds the scope of this paper. The whole process of detection is described in [32].

\section{Methodology of eVAluation}

As mentioned earlier, the aim of this paper is to evaluate the suitability of selected interest point detectors for ultrasound images. Robust interest points have to be independent of the noise in ultrasound images and stable under brightness variation.

We chose three interest point detectors described in section 2 for comparison. Firstly, the most appropriate setup of parameters of the detectors was found so that they detect a similar number of interest points in the images. This setup was performed partly according to experience and partly with the utilization of grid search. This process will be described in detail in section 3.1.

After the initial setup of detectors is completed, it is possible to compare the performance of selected detectors. Repeatability rate was chosen as a measure for comparison of the detectors. Repeatability rate is defined as the number of points repeated between two images (one image is original, the second one is modified) with respect to the total number of detected points. The experiment is based on gradual degradation of image by noise and measurement of repeatability. The better a detector performs the higher is the value of repeatability. The experiment is further described in section 3.5.

\subsection{Initial setup of interest point detectors}

It was necessary to set parameters of the detectors to detect a similar number of interest points before the comparison itself. We combined two approaches to set the parameters. Firstly, we identified parameters that affect and do not affect the number of detected points. From the ones that influence the number of detected points, we selected some of the parameters and applied the grid search algorithm to find their suitable values.

The grid search represents one of the so called brute-force search algorithms. These algorithms search the parameter space by trying all possible combinations of the parameters. A step has to be defined for each parameter. The step and the number of parameters influence the computational demands of this algorithm rapidly. The step is therefore a trade-off between speed and accuracy. Initially, we ran the algorithm with large step and selected intervals which were searched exhaustively in subsequent runs. The obtained results can be found in section 5.2.

\subsection{Repeatability rate}

We used repeatability rate to evaluate the detectors. Repeatability rate is described in [24] in detail. It is a measure that directly evaluates the quality of detected points which is applicable to any type of scene. Let us assume that we have two versions of the image acquired under different conditions. Repeatability rate compares the geometrical stability of the detected interest points in these two images. Furthermore, no specific high-level interpretation of the features is needed to evaluate repeatability. Repeatability rate $r_{i}(\varepsilon)$ is defined as

$$
r_{i}(\varepsilon)=\left|\frac{R_{i}(\varepsilon)}{\min \left(n_{1}, n_{i}\right)}\right|,
$$

where $R_{i}(\varepsilon)$ equals to the number of point pairs $\left(x_{1} ; x_{i}\right)$ which correspond within an $\varepsilon$-neighborhood and $n_{1}=\left|\left\{\tilde{x}_{1}\right\}\right|$ and $n_{i}=\left|\left\{\tilde{x}_{i}\right\}\right|$ are the number of points detected in the common part of images $I_{1}$ and $I_{i}$. It can be proved that the resulting value ranges between zero and one and therefore is often expressed in percentage. We set $\varepsilon$ to 0.5 in our experiment.

\subsection{Noise models}

We evaluated repeatability rate for images which were artificially corrupted by noise. Specifically, we used two types of noise: the additive Gaussian noise and the speckle noise which is specific for ultrasound images.

The noise model of the additive Gaussian noise is very simple and can be expressed as

$$
I^{\prime}(\mathbf{x})=I(\mathbf{x})+n
$$

where $I(\mathbf{x})$ refers to the original image, $n$ represents the noise and $I^{\prime}(\mathbf{x})$ is the image corrupted by noise. The values of $I(\mathbf{x})$ are expected to be floating point numbers in the range $\langle 0,1\rangle$. The values of the noise follow the normal distribution with zero mean and variance $\left(\sigma^{2}\right)$ which is changed during the experiment. Values of $I^{\prime}(\mathbf{x})$ were clipped to range $\langle 0,1\rangle$ after the noise was added.

The speckle noise description differs slightly. It can be described by the following equation:

$$
I^{\prime}(\mathbf{x})=I(\mathbf{x})+u_{0} I(\mathbf{x}),
$$

where $u_{0}$ is the noise. It can be directly seen that it is a multiplicative noise model. The noise in this case follows the uniform distribution with zero mean and variance which was adjusted during the experiment. 


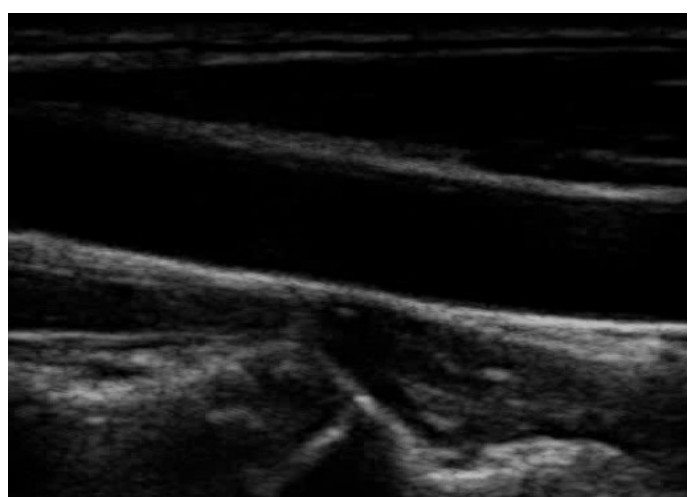

Fig. 1: Sample image from the database.

\subsection{Image database}

The image database contains $84 \mathrm{~B}$-mode ultrasound images of common carotid artery (CCA) in longitudinal section (Fig. 1). The database contains images of CCA of ten volunteers (mean age $27.5 \pm 3.5$ years) with different weight (mean weight $76.5 \pm 9.7 \mathrm{~kg}$ ). Images were acquired with Sonix OP ultrasound scanner with different set-up of depth, gain, time gain compensation (TGC) curve and different linear array transducers. The resolution of images is approximately $390 \times$ $330 \mathrm{px}$. The exact resolution depends on the set-up of the ultrasound scanner. Two different linear array transducers with different frequencies $(10 \mathrm{MHz}$ and $14 \mathrm{MHz}$ ) were used. These frequencies were chosen because of their suitability for superficial organ imaging. All images were taken by specialists with five year experience with scanning of arteries. Images were captured in accordance to the standard protocol [33] with patients lying in the supine position and with the neck rotated to the left side while the right CCA was examined.

The database can be downloaded from the SPLab web page $^{1}$. As far as we know, this database is the only one freely available database of ultrasound images of common carotid artery.

\subsection{Process of evaluation}

The comparison of interest point detectors consists of three experiments. First two experiments focus on the measurement of repeatability in images corrupted by varying noise. The third experiment measures repeatability in images with changing brightness. The flowcharts describing all three processes are depicted in Fig. 2. The only difference between the three figures is in one block that performs the modification which is specific to each experiment.

The speckle noise was added to the input image data set during the first experiment. The experiment was conducted according to the flow chart shown in Fig. 2a. The second experiment differs only in the type of noise which was added to the image. It was additive Gaussian noise in this case. A flowchart describing the process can be found in Fig. 2 b. The variance of the noise was changed from 0.0 to 1.0 with

\footnotetext{
${ }^{1}$ http://splab.cz/en/download/databaze/ultrasound
}

quadratic scale during both experiments. The third experiment examined the sensitivity of detectors to the change of brightness in ultrasound images. The parameter $k$, which transforms the gray scale according to $I^{\prime}(\mathbf{x})=k I(\mathbf{x})$, was changed during this experiment. Steps performed in this experiment are depicted in a flowchart in Fig. 2c.

\section{IMPLEMENTATION}

Using Matlab to implement the algorithms that are being proposed in papers has become almost standard. We are fully aware of many advantages that Matlab brings but unlike many other research teams that use Matlab, we used a novel approach to implement the whole process of evaluation of the detectors. We built the whole process on top of RapidMiner ${ }^{2}$ which is a popular tool meant for data mining. It is designed to process numerical data that can be imported in a number of different formats (xls, csv, etc.). RapidMiner offers more than 100 learning algorithms that can be applied to the data.

Thanks to the simple extension mechanism new plugins can be added to RapidMiner. The plugins can either implement new data mining algorithms or provide means to extract numerical data from other media (like images). The wide range of data mining methods available in RapidMiner can be then used to analyze the extracted data.

The simplicity of the extension mechanism was fully exploited by members of SPLab at the Brno University of Technology who have recently developed an extension, called IMMI (IMage MIning) [34]. The extension offers over 140 algorithms that cover the basic as well as some advanced image processing techniques.

Nonetheless, methods for corrupting the image by noise, change of brightness of the image, interest point detection and evaluation of interest point detectors had to be added.

The Harris-Laplace detector was implemented according to the paper by K. Mikolajczyk and C. Schmid [25]. The DoG detector was also implemented according to the original paper by D.G.Lowe [32]. The method is protected under US patent, ${ }^{3}$ but it is free for individual research use. The original implementation of the Fast Hessian detector (used in the SURF (Speeded Up Robust Features) detector and descriptor) is closed-source and it is not suitable for research. However, there is a number of open-source implementations of the SURF algorithm but they differ from the original one. An evaluation of some of the open-source implementations is available in [35]. Our implementation is based on the OpenSURF library [36] which was ported to Java programming language.

One of the main contributions of this paper is the implementation of these functions into the IMMI extension. The

\footnotetext{
${ }^{2}$ http://rapid-i.com

${ }^{3}$ Method and apparatus for identifying scale invariant features in an image and use of same for locating an object in an image David G. Lowe, US Patent 6,711,293 (March 23, 2004). Provisional application filed March 8, 1999. Asignee: The University of British Columbia.
} 


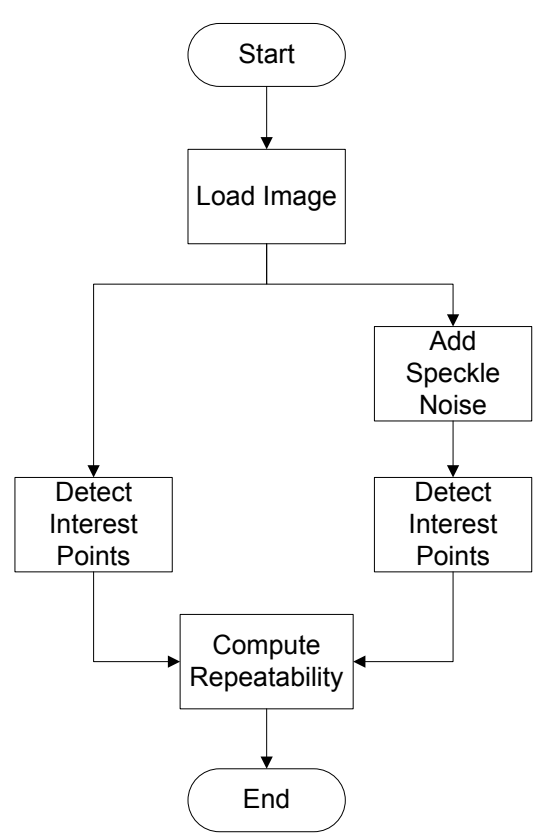

(a)

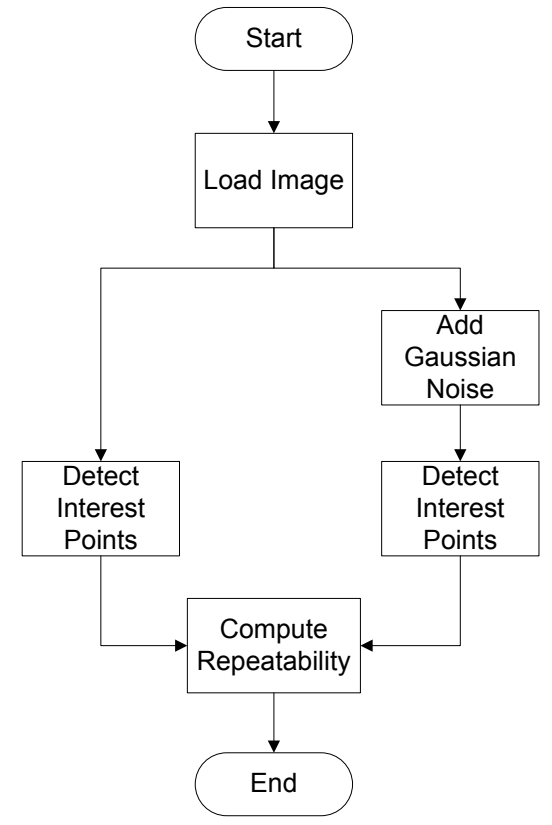

(b)

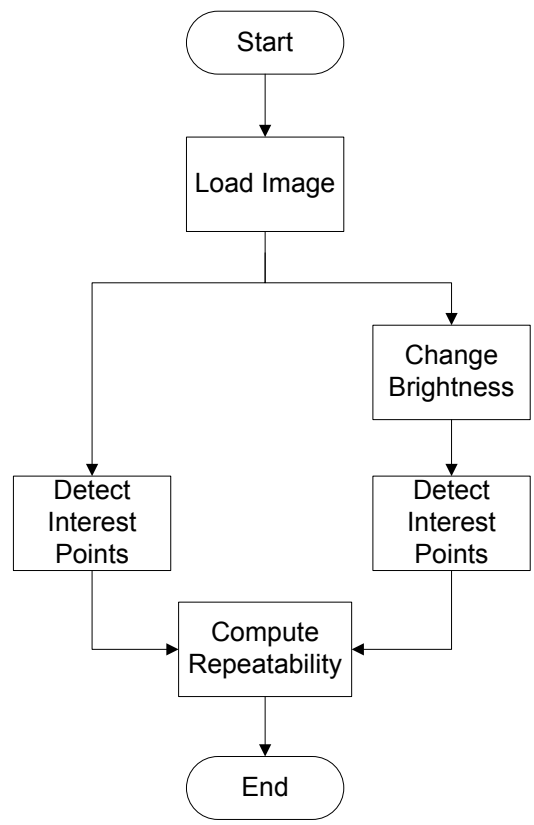

(c)

Fig. 2: Flow charts for three experiments.

IMMI extension is freely available for non-commercial purposes and can be downloaded from the project home page 4 .

\section{RESUlTS}

\subsection{Selected setup of interest point detectors}

As mentioned above, certain parameters were chosen according to recommended settings and experience. The rest of the parameters was set using the grid search method. The grid search method gradually adjusts the setup of the parameters of detectors and measures the number of detected points. The dependences of the number of detected points with changing setup of parameters for different detectors are shown in Fig. 3, Fig. 4 and Fig. 5. The main presumption when choosing the most suitable setup of parameters was based on the fact, that detectors are comparable, if they detect similar count of interest points (in our case, approximately 23000 interest points in 84 ultrasound images). The final parameter setup for each detector is summarized in Tab. 1 and the number of detected points is in Tab. 2.

The octave parameter defines the number of steps in each octave (doubling of $\sigma=$ downscaling the image). The scale parameter determines the maximum number of scales that will be searched for interest points. It influences the width and $\sigma$ of the Gaussian kernel which is used to downscale the image. The threshold parameter serves to reject points with weak response. The initial sigma parameter (as the name implies) is used as the initial $\sigma$ of the Gaussian kernel.

\footnotetext{
${ }^{4}$ http://splab.cz/en/research/data-mining/articles
}

Table 1: The final parameter setup for each interest point detectors.

\begin{tabular}{lll}
\hline Parameter & Type of choice & Value \\
\hline \multirow{2}{*}{ Octaves } & Fast Hessian & \\
Threshold & GS (Grid search) & 1 \\
& GS & 6.3 \\
Threshold & Harris-Laplace & \\
Octaves & GS & 0 \\
Scales & GS & 5 \\
& Difference of Gaussian & 16 \\
Octaves & GS & 3 \\
Initial sigma & GS & 1.6
\end{tabular}

Table 2: Number of points detected by each detector.

\begin{tabular}{ll}
\hline Detector & Number of points \\
\hline Fast Hessian & 22750 \\
Harris-Laplace & 20309 \\
Difference of Gaussian detector & 23764 \\
\hline
\end{tabular}

Examples of points detected by the detectors (with setup that was described above) in a sample image from the database are presented in Fig. 6. The size of the circles corresponds to the strength of the response of the algorithm to the interest point. Points detected in an unmodified image are depicted in the first row. The second row shows the same image corrupted with additive noise of variance equal to 0.12 and points detected by the detectors in this image. It can be 


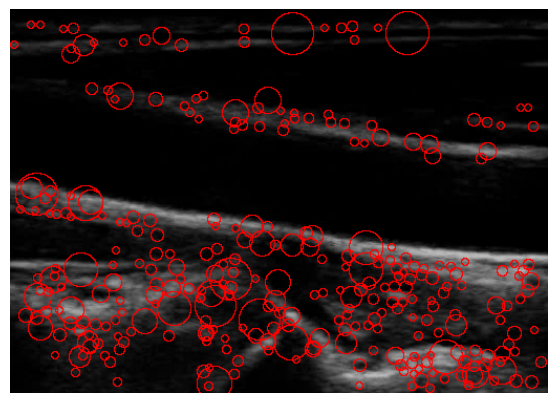

(a) Difference of Gaussian detector

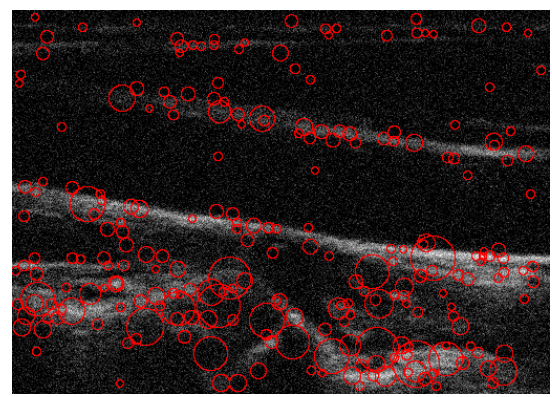

(d) Difference of Gaussian detector

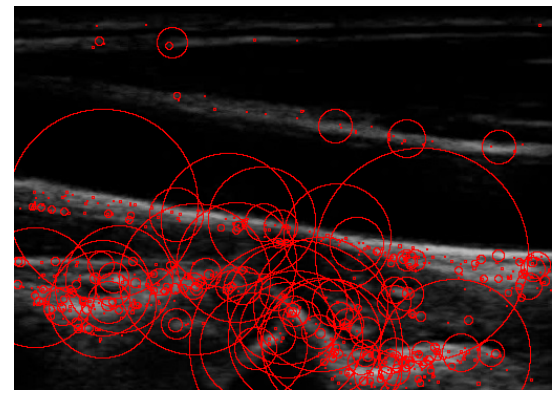

(b) Harris-Laplace detector

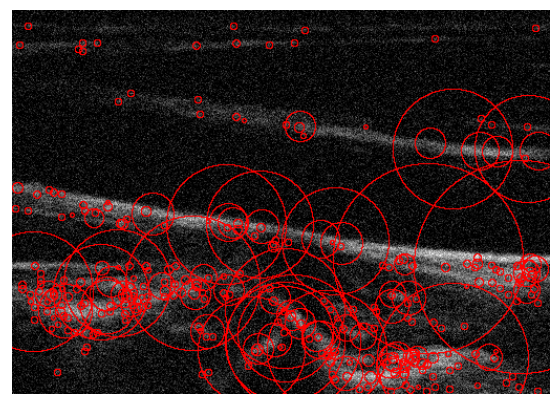

(e) Harris-Laplace detector

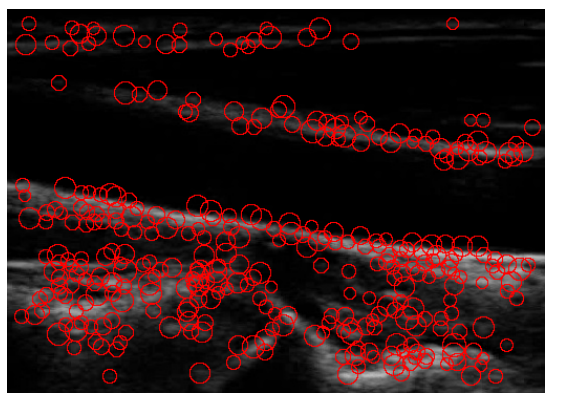

(c) Fast Hessian detector

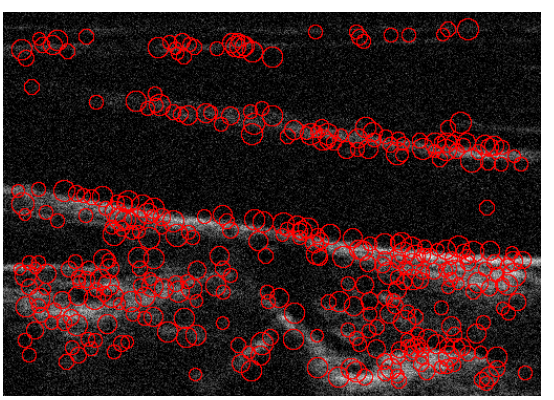

(f) Fast Hessian detector

Fig. 6: First row: Sample image with detected interest points. Second row: Sample image corrupted with additive noise $(\sigma=0.12)$ with detected interest points

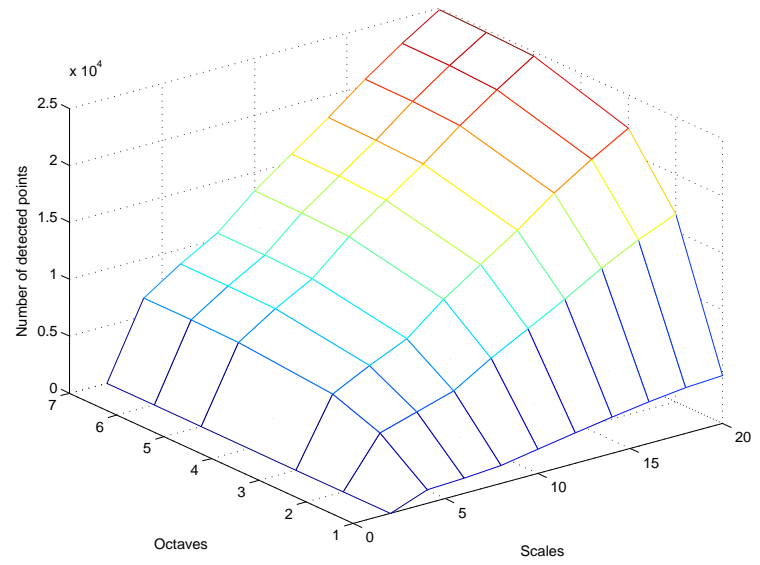

Fig. 3: Number of points detected by Harris-Laplace detector for different parameter setups.

observed that in noised image the number of detected interest points increases because the previously uniform areas now contain more details that are considered as interest points by the detectors.

\subsection{Dependence of repeatability rate on image modification}

The dependence of repeatability on the amount of speckle noise for tested detectors is depicted in Fig. 7. The results in Fig. 7 show that most resistant to speckle noise is the HarrisLaplace detector, which achieves good level of repeatability even if the level of added noise is significant.

Figure 8 shows the results of the second experiment during which the Gaussian noise was added to the images. The

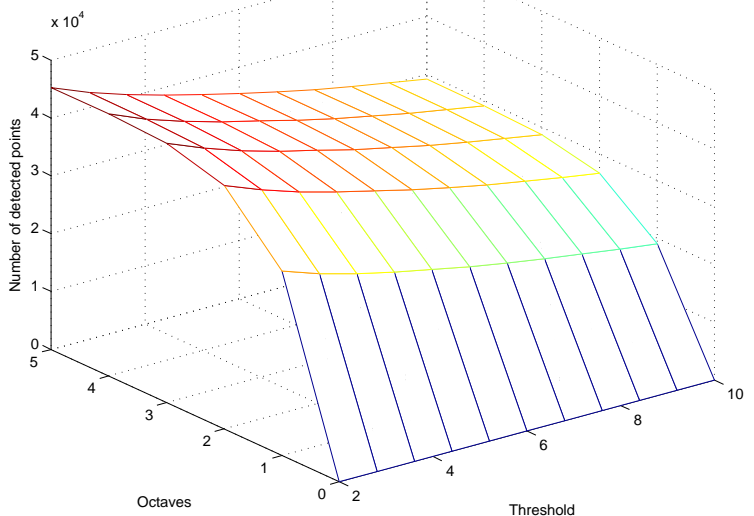

Fig. 4: Number of points detected by Fast Hessian detector for different parameter setups.

Harris-Laplace detector achieves best results also in this experiment.

The results obtained in the experiment that changed the brightness of the image can be found in Fig. 9. The best detector for slightly brightened images $(k \in\langle 1,1.5\rangle)$ is again Harris-Laplace. However, the Harris-Laplace detector achieves worse results for more brightened images $(k>1.5)$ and darkened images $(k<1)$

\section{DISCUSSION}

The results suggest that the most suitable interest point detector for US images is the Harris-Laplace detector. It outperforms the other detectors even in cases when the images 


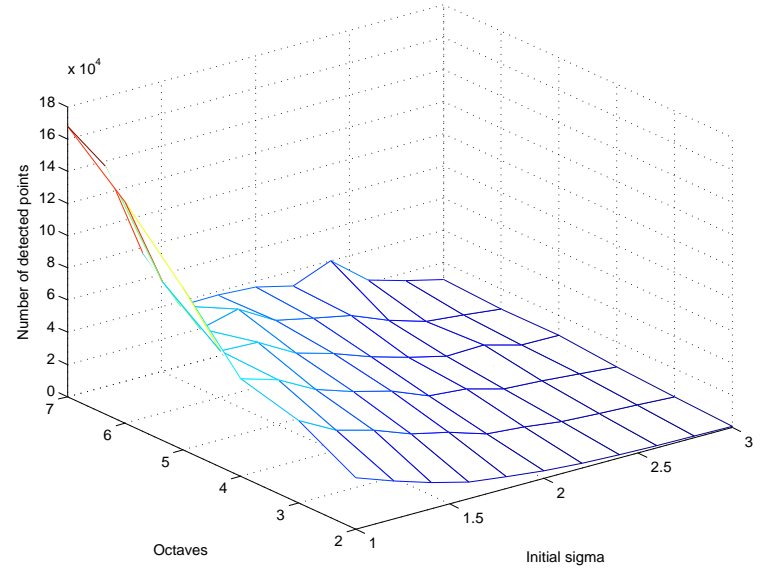

Fig. 5: Number of points detected by Difference of Gaussian detector for different parameter setups.

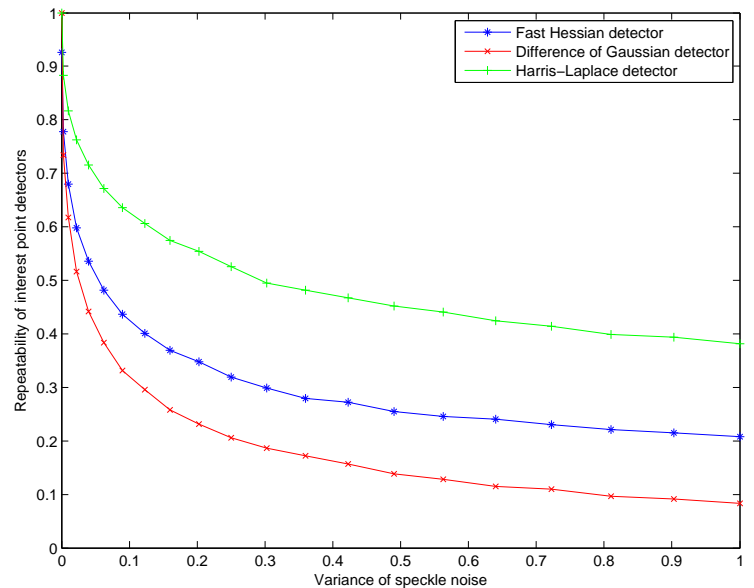

Fig. 7: Repeatability of detectors for different level of speckle noise.

are significantly corrupted by noise. Nonetheless, the HarrisLaplace detector has considerably higher computational complexity than the DoG and the Fast Hessian detector. The reason for this is that the matrix $M$ contains only the first order derivatives of the scale-space function $L_{x}\left(\mathbf{x}, \sigma_{n}\right)$ and $L_{y}\left(\mathbf{x}, \sigma_{n}\right)$ and not the second order derivatives (see equation 4 and compare it with equations 8 and 9). The second order derivatives $L_{x x}\left(\mathbf{x}, \sigma_{n}\right)$ and $L_{y y}\left(\mathbf{x}, \sigma_{n}\right)$, which are needed for selecting the scale-invariant points, have to be therefore computed in a separate step which results in a lower processing speed. A comparison of the Harris-Laplace and DoG detectors in terms of computational complexity can be found in [25]. From this paper follows that the run time of the Harris-Laplace detector is ten times higher than in case of the DoG detector which corresponds with our results. The computational complexity of the Harris-Laplace detector can be further reduced as proposed by Mikolajczyk and Schmid in the same work [25] which shortens the run time to approximately a double of the value achieved by the DoG detector. The authors of the Fast Hessian detector claim that their detector outperforms the DoG detector in terms of the run time more than three times [30]. We can conclude that the Fast Hessian detector has lowest complexity, the DoG is in the middle and the Harris-Laplace

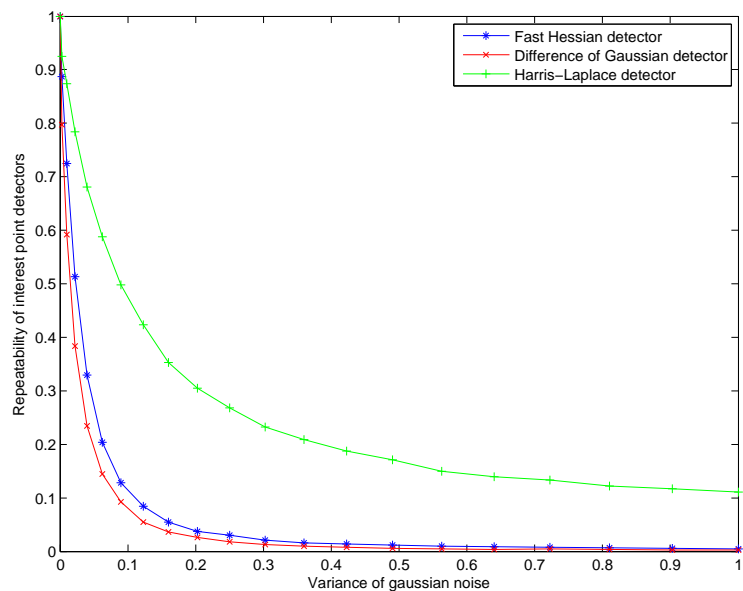

Fig. 8: Repeatability of detectors for different level of Gaussian noise.

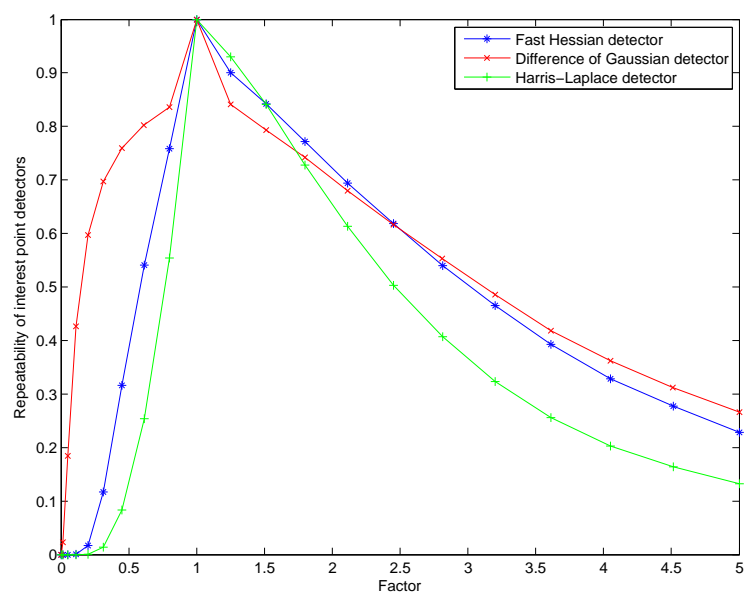

Fig. 9: Repeatability of detectors for different changes of brightness. If factor $k>1$ the image is brightened, if factor $k<1$ the image is darkened.

detector has the highest complexity.

We can compare the results from [25] and [26] with the experiment where the brightness of the image was changed and the repeatability of detectors was measured. Different datasets are used for evaluation in the aforementioned papers. In the former study the authors use their own dataset while two databases popular in the image processing community are used in the latter one. It is also important to mention that the former study does not evaluate the same three detectors as we used and only the results of the Harris detector can be indirectly compared with the results of the HarrisLaplace detector which is a scale-invariant version of the Harris detector. The Harris detector achieved better results than its scale-invariant counterpart which means that the HarrisLaplace detector tends to favor points that are scale invariant over the ones that are stable under change of brightness. In [26], the Fast Hessian and DoG detectors notably outperformed the Harris-Laplace detector especially when the image was darkened. These results were more or less confirmed also in our study. Overall results of the detectors were bet- 
ter when tested on standard (meaning not ultrasound) images. This may be explained by the fact that the US images contain higher level of specific noise which is in some cases falsely detected as interest point. The authors in [27] do not use the repeatability rate as the measure, therefore, it is not possible to directly compare their results with ours.

\section{CONCLUSION}

This paper compares suitability of three interest point detectors for use in US images. Interest point detection is a necessary step preceding medical examinations that are based on speckle tracking such as continual measurement of lumen diameter, arterial stiffness and arterial compliance. The tracked interest points have to be invariant to noise as much as possible for high accuracy of long-lasting measurement. We performed three experiments that measure repeatability of detectors in varying noise (Gaussian and speckle) and changes in brightness.

- The first two experiments examined the stability of the detectors under changing variance of noise of different types. The results show that the most robust is the HarrisLaplace detector whose repeatability does not drop so steeply when compared to the other ones.

- The third experiment focuses on change of brightness in the images. The Harris-Laplace detector did well also in this experiment. It achieved best results for values of $k$ in range $\langle 1,1.5\rangle$. Nevertheless, DoG and Fast Hessian outperformed Harris-Laplace when the images were darkened and also for values of $k$ larger than 1.5.

All the experiments were conducted with utilization of the IMMI extension of RapidMiner [34]. New processes for finding the optimal setup of parameters and evaluation of the quality of the interest point detectors were designed and published in this paper.

Additional contributions of this paper reside in acquisition and publishing of a new ultrasound image database and in the implementation of all necessary operators into the IMMI extension of RapidMiner. As the IMMI extension and the image database are freely available, all tested interest point detectors, as well as other operators, can be utilized by other research teams.

A possible direction for future work is to test other interest point detectors such as the Harris corner detector [29] or the salient point detectors described in [21, 22].

\section{ACKNOWLEDGEMENTS}

The described research was performed in laboratories supported by the SIX project; the registration number CZ.1.05/2.1.00/03.0072, the operational program Research and Development for Innovation. The research was also supported by the project FEKT-S-11-17 Research of sophisticated methods for digital audio and image signal processing sponsored by the Brno University of Technology.

\section{REFERENCES}

[1] Acharya, R., Wasserman, R., Stevens, J., Hinojosa, C. (1995). Biomedical imaging modalities: a tutorial. Computerized Medical Imaging and Graphics 19(1), 3-25.

[2] Tay, P. C., Acton, S. T., Hossack, J. A. (2011). A wavelet thresholding method to reduce ultrasound artifacts. Computerized Medical Imaging and Graphics $35(1), 42-50$.

[3] Sun, Q., Hossack, J. A., Tang, J., Acton, S. T. (2004). Speckle reducing anisotropic diffusion for $3 \mathrm{~d}$ ultrasound images. Computerized Medical Imaging and Graphics 28(8), 461-470.

[4] Notomi, Y., Lysyansky, P., Setser, R. M., Shiota, T., Popovic, Z. B., Martin-Miklovic, M. G., Weaver, J. A., Oryszak, S. J., Greenberg, N. L., White, R. D., Thomas, J. D. (2005). Measurement of ventricular torsion by two-dimensional ultrasound speckle tracking imaging. Journal of the American College of Cardiology 45(12), 2034-2041.

[5] Jayasree, V. K., Sandhya, T. V., Radhakrishnan, P. (2008). Non-invasive studies on age related parameters using a blood volume pulse sensor. Measurement Science Review 8, 82-86.

[6] Hlimonenko, I., Meigas, K., Vahisalu, R. (2003). Waveform analysis of peripheral pulse wave detected in the finger tip with photoplethysmograph. Measurement Science Review 3, 49-52.

[7] Tuytelaars, T., Mikolajczyk, K. (2008). Local Invariant Feature Detectors: A Survey. Hanover, MA, USA: Now Publishers Inc.

[8] Mital, P., Smith, T., Hill, R., Henderson, J. (2011). Clustering of gaze during dynamic scene viewing is predicted by motion. Cognitive Computation 3, 5-24. 10.1007/s12559-010-9074-z.

[9] McEachen, J. C., I., Duncan, J. S. (1997). Shape-based tracking of left ventricular wall motion. IEEE Trans. Med. Imag. 16(3), 270-283.

[10] Mailloux, G. E., Bleau, A., Bertrand, M., Petitclerc, R. (1987). Computer analysis of heart motion from twodimensional echocardiograms. IEEE Trans. Biomed. Eng. BME-34(5), 356-364.

[11] Friedland, N., Adam, D. (1989). Automatic ventricular cavity boundary detection from sequential ultrasound images using simulated annealing. IEEE Trans. Med. Imag. 8(4), 344-353.

[12] Cinthio, M., Ahlgren, A. R., Jansson, T., Eriksson, A., Persson, H. W., Lindstrom, K. (2005). Evaluation of an ultrasonic echo-tracking method for measurements of arterial wall movements in two dimensions. IEEE Trans. Ultrason., Ferroelectr., Freq. Control 52(8), 1300-1311.

[13] Cinthio, M., Ahlgren, A. R., Bergkvist, J., Jansson, T., Persson, H. W., Lindstrom, K. (2006). Longitudinal movements and resulting shear strain of the arterial wall. 
American Journal of Physiology - Heart and Circulatory Physiology 291(1), H394-H402.

[14] Andrea, Giachetti (1998). On-line analysis of echocardiographic image sequences. Medical Image Analysis 2(3), 261-284.

[15] Riha, K., Potucek, I. (2009). The sequential detection of artery sectional area using optical flow technique. In: Proceedings of The 8th WSEAS International Conference on CIRCUITS, SYSTEMS, ELECTRONICS, CONTROL \& SIGNAL PROCESSING. pp. 222-226.

[16] Riha, K., Benes, R. (2011). Testing of methods for artery section area detection. In: Proceedings of the 10th WSEAS international conference on Telecommunications and informatics and microelectronics, nanoelectronics, optoelectronics, and WSEAS international conference on Signal processing. Stevens Point, Wisconsin, USA. pp. 184-187.

[17] Kelly, A. S., Kaiser, D. R., Dengel, D. R., Bank, A. J. (2004). Comparison of b-mode and echo tracking methods of assessing flow-mediated dilation. Ultrasound in Medicine \&amp; Biology 30(11), 1447-1449.

[18] Liao, J., Bettmann, M., Sandor, T., Tucker, J., Coleman, S., Creager, M. (1991). Differential impairment of vasodilator responsiveness of peripheral resistance and conduit vessels in humans with atherosclerosis. Circulation Research 68(4), 1027-1034.

[19] Persson, M., Ahlgren, A. R., Jansson, T., Eriksson, A., Persson, H. W., Lindstrom, K. (2003). A new noninvasive ultrasonic method for simultaneous measurements of longitudinal and radial arterial wall movements: first in vivo trial. Clinical Physiology and Functional Imaging 23(5), 247-251.

[20] Nishizawa, O., Matsuzaki, A., Kohama, T., Suzuki, T., Noto, H. (1985). Noninvasive screening procedure for evaluation of bladder emptying function. Tohoku J. Exp. Med. 147(4), 421-525.

[21] Sebe, N., Lew, M. S. (2003). Comparing salient point detectors. Pattern Recognition Letters 24, 89-96.

[22] Pedersen, K. S., Loog, M., van Dorst, P. (2007). Salient point and scale detection by minimum likelihood. In: Gaussian Processes in Practice. pp. 59-72.

[23] Pedersen, K. S. (2003). Properties of brownian image models in scale-space. In: Griffin, L. D., Lillholm, M. (eds.) Scale-Space, Vol. 2695 of Lecture Notes in Computer Science. pp. 281-296.

[24] Schmid, C., Mohr, R., Bauckhage, C. (2000). Evaluation of interest point detectors. Int. J. Comput. Vision 37, 151-172.

[25] Mikolajczyk, K., Schmid, C. (2004). Scale \& affine invariant interest point detectors. Int. J. Comput. Vision 60(1), 63-86.

[26] Zukal, M., Cika, P., Burget, R. (2011). Evaluation of interest point detectors for scenes with changing light- ening conditions. In: Telecommunications and Signal Processing (TSP) 2011, 34th International Conference. pp. 579-583.

[27] Tissainayagam, P., Suter, D. (2004). Assessing the performance of corner detectors for point feature tracking applications. Image and Vision Computing 22, 663-679.

[28] Lindeberg, T. (1994). Scale-space theory: A basic tool for analysing structures at different scales. Journal of Applied Statistics, 224-270.

[29] Harris, C., Stephens, M. (1988). A combined corner and edge detection. In: Proceedings of The Fourth Alvey Vision Conference. pp. 147-151.

[30] Bay, H., Tuytelaars, T., Gool, L. V. (2006). Surf: Speeded up robust features. In: In ECCV. pp. 404-417.

[31] Viola, P., Jones, M. (2002). Robust real-time object detection. International Journal of Computer Vision 57(2), 137-154.

[32] Lowe, D. G. (2004). Distinctive image features from scale-invariant keypoints. Int. J. Comput. Vision 60(2), 91-110.

[33] Casella, I., Presti, C., Porta, R., Sabbag, C., Bosch, M., Yamazaki, Y. (2008). A practical protocol to measure common carotid artery intima-media thickness. Clinics 63(4), 515-20.

[34] Burget, R., Karasek, J., Smekal, Z., Uher, V., Dostal, O. (2010). Rapidminer image processing extension: A platform for collaborative research. In: International Conference on Telecommunications and Signal Processing. Baden, Austria. pp. 114-118.

[35] Gossow, D., Decker, P., Paulus, D. (2010). An evaluation of open source surf implementations. In: RobuCup. pp. 169-179.

[36] Evans, C. (2009). Notes on the opensurf library. Technical report, University of Bristol.

Received January 12, 2013. Accepted December 10, 2013. 